\title{
Biochemical changes causes lack of bioluminescence in fruiting bodies of Armillaria
}

\section{Puzyr AP, Medvedeva SE and Bondar VS}

Institute of Biophysics, Siberian Branch of Russian Academy of Science, Federal Research Center "Krasnoyarsk Science Center SB RAS”, Akademgorodok, 660036 Krasnoyarsk, Russia

Puzyr AP, Medvedeva SE, Bondar VS 2017 - Biochemical changes causes lack of bioluminescence in fruiting bodies of Armillaria. Mycosphere 8(1), 9-17, Doi 10.5943/mycosphere/8/1/2

\begin{abstract}
Mycelium of Armillaria species exhibit bioluminescence in nature and when cultivated on artificial nutrient media. However, fruiting bodies do not emit visible light. The present study investigates biochemical changes which cause this phenomenon. Light emission was studied in experiments with mixtures of cold and hot extracts of the luminous mycelium of Armillaria borealis IBSO 2328 and nonluminous fruiting bodies of this fungus and an unidentified species of the genus (Armillaria sp.). Hot extracts of fruiting bodies of the nonluminous Pholiota squarrosa were used as the substrate analog of the luminescent reaction, as previously this fungus had been found to contain a high amount of this substance. Control experiments showed that cold extracts of A. borealis IBSO 2328 mycelium contained enzymes for the luminescent reaction, which is initiated after addition hot extracts of $P$. squarrosa fruiting bodies. Parallel experiments with extracts of the fruiting bodies of Armillaria showed that: (i) - cold extracts did not contain enzymes of the luminescent reaction or contain very small amounts of these enzymes and (ii) - hot extracts did not contain substrate of the luminescent reaction. Thus, the reason why fruiting bodies of Armillaria do not emit light is that they do not contain components required for visible luminescence. The study discusses possible causes why the enzymes and substrate of the luminescent reaction are not synthesized in fruiting bodies of Armillaria.
\end{abstract}

Key words - luminous mycelia - nonluminous fruiting bodies of fungus - enzymes and substrate of luminescent reaction - kinetics of luminescence

\section{Introduction}

Many higher fungi emit visible light, i.e. exhibit bioluminescence (Harvey 1952, 1957, Johnson \& Haneda 1966, Shimomura 2006, Desjardin et al. 2008). Over 80 species of luminous basidiomycetes have been found in different regions of the world (Desjardin et al. 2008, Vydryakova et al. 2009, Chew et al. 2015, Mihail 2015). In some species, luminescence occurs in the whole fruiting body (Van 2009, Brandl 2011, Vydryakova et al. 2012, Oliveira et al. 2015). In other species, only caps or stems emit light (Desjardin et al. 2007, 2008, Teranishi 2016 a, b). It is well known that fruiting bodies of basidiomycetes of the genus Armillaria are not able to emit visible light (Harvey 1952, Wassink 1978, Desjardin et al. 2008, Mihail 2013, 2015). Luminescence occurs only in mycelium, growing on the natural substrate (wood) or artificial substrates (nutrient medium) (Shimomura 2006, Mihail \& Bruhn 2007, Medvedeva et al. 2014). We have not found any published studies addressing biochemical causes of this phenomenon. 
In the present paper, we report a study that explains and describes the reasons for the lack of luminescence in the fruiting bodies of basidiomycetes of the genus Armillaria.

\section{Materials \& Methods}

In this study, we used fruiting bodies of Armillaria borealis and an Armillaria sp., collected around Krasnoyarsk (Russia) in September 2016. The biomass of fruiting bodies was used to prepare cold and hot extracts, which were analyzed for the presence of the enzymes and components the luminescent reaction. Cold extracts were also prepared from the biomass of luminous mycelium of Armillaria borealis strain IBSO 2328 from the Collection of the cultures of microorganisms CCIBSO 836 in the Institute of Biophysics SB RAS. As a substrate analog of the luminescence reaction, we used hot extract of the fruiting bodies of the nonluminous Pholiota squarrosa (Purtov et al. 2015, Puzyr et al. 2016).

Biomass of the luminous mycelium of $A$. borealis was prepared by growing the fungus in submerged culture. Finely crushed mycelium of A. borealis strain IBSO 2328 which had been grown on solid medium in Petri-dishes was used as the inoculum. Mycelium pellets were grown in liquid PDB nutrient medium (potato extract - $200 \mathrm{~g} / \mathrm{L}$, dextrose $-20 \mathrm{~g} / \mathrm{L}$ ). The fungus was cultivated in $300-\mathrm{ml}$ flasks containing $100 \mathrm{ml}$ of nutrient medium. Cultivation was conducted for $16-20 \mathrm{~d}$ at a temperature of $24^{\circ} \mathrm{C}$ under constant agitation at 160-180 rpm, using a Max Q 4000 incubating shaker (Thermo Scientific, U.S.). PDB nutrient medium was purchased from HiMedia Laboratory (India).

The biomass of $A$. borealis mycelium pellets was taken out of the nutrient medium and rinsed in deionized water to remove residual nutrient medium and metabolites. Deionized water was produced in a Milli-Q system (Millipore, U.S.). The rinsed pellets were transferred into a larger volume of deionized water and incubated for $24 \mathrm{~h}$ with the air constantly bubbling through the water. It is well known (Mori et al. 2011, Bondar et al. 2013, Mogilnaya et al. 2015, 2016) that incubation of the biomass of luminous fungi in water increases their luminescence level. Prior to using A. borealis, Armillaria sp., and P. squarrosa fruiting bodies to prepare cold and hot extracts, they were thoroughly rinsed in distilled water to remove contaminants. The procedure of preparing cold and hot extracts from the fungal biomass (mycelium and fruiting bodies) was described in detail elsewhere (Puzyr et al. 2016). All extracts were frozen at $-30^{\circ} \mathrm{C}$ in a Sanyo Biomedical Freezer, model MDF - U333 (SANYO Electric Co., Ltd., Japan) and stored at this temperature until used. Before experiments, the extracts stored in MCT-200-C microtubes (Axygen Scientific, Inc., U.S.) were thawed at room temperature $\left(23-25^{\circ} \mathrm{C}\right)$ and placed into a glass of ice where they were kept throughout the study.

Samples of cold and hot extracts were mixed and the levels of light emission were measured to determine the presence of enzymes and substrates of the luminescent reaction in the extracts. The amplitude and dynamics of the light signal were measured using a Glomax ${ }^{\circledR}$ 20/20 luminometer (Promega BioSystems Sunnyvale, Inc., U.S.). The measurements were conducted as follows. A sample of cold extract $(200 \mu \mathrm{l})$ was poured into MCT-200-C microtubes which were placed in a luminometer; then, it was supplemented with $5 \mu 110 \mathrm{mM} \mathrm{NADPH}$ and $5 \mu 1$ hot extract to trigger the luminescent reaction. Luminescence was monitored at a rate of one measurement per sec. Light emission intensity was expressed as relative light units (RLU) per sec.

Images of the appearance and luminescence of mycelium samples of A. borealis growing under natural conditions and in submerged culture and the appearance of its fruiting bodies were prepared using a GelDoc XR Imaging System (Bio-Rad Laboratories, Inc., U.S.). The conditions for detecting the luminescent signal of mycelium samples were as follows (Puzyr et al. 2016): the diaphragm - open maximally, the time of signal accumulation - to $300 \mathrm{sec}$. At a low luminescence level, the image was enhanced by using the Transform function of the software: gamma 4.00, low 60021. 


\section{Results}

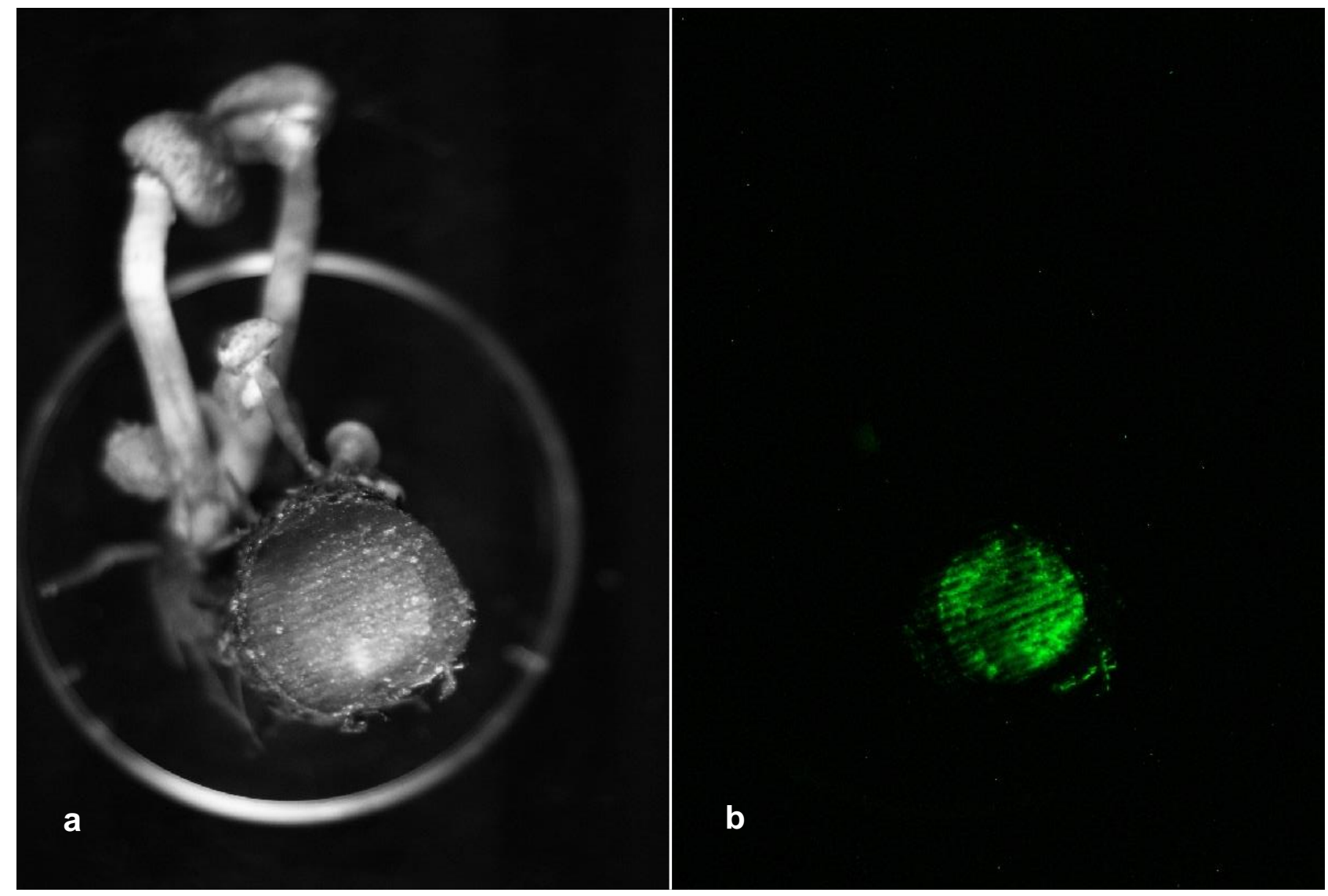

Fig. 1 - The image of a wood sample with A. borealis fruiting bodies: a. visible light. b. luminescence of mycelium on the cross section of the wood sample. Images prepared using a GelDoc XR Imaging System.
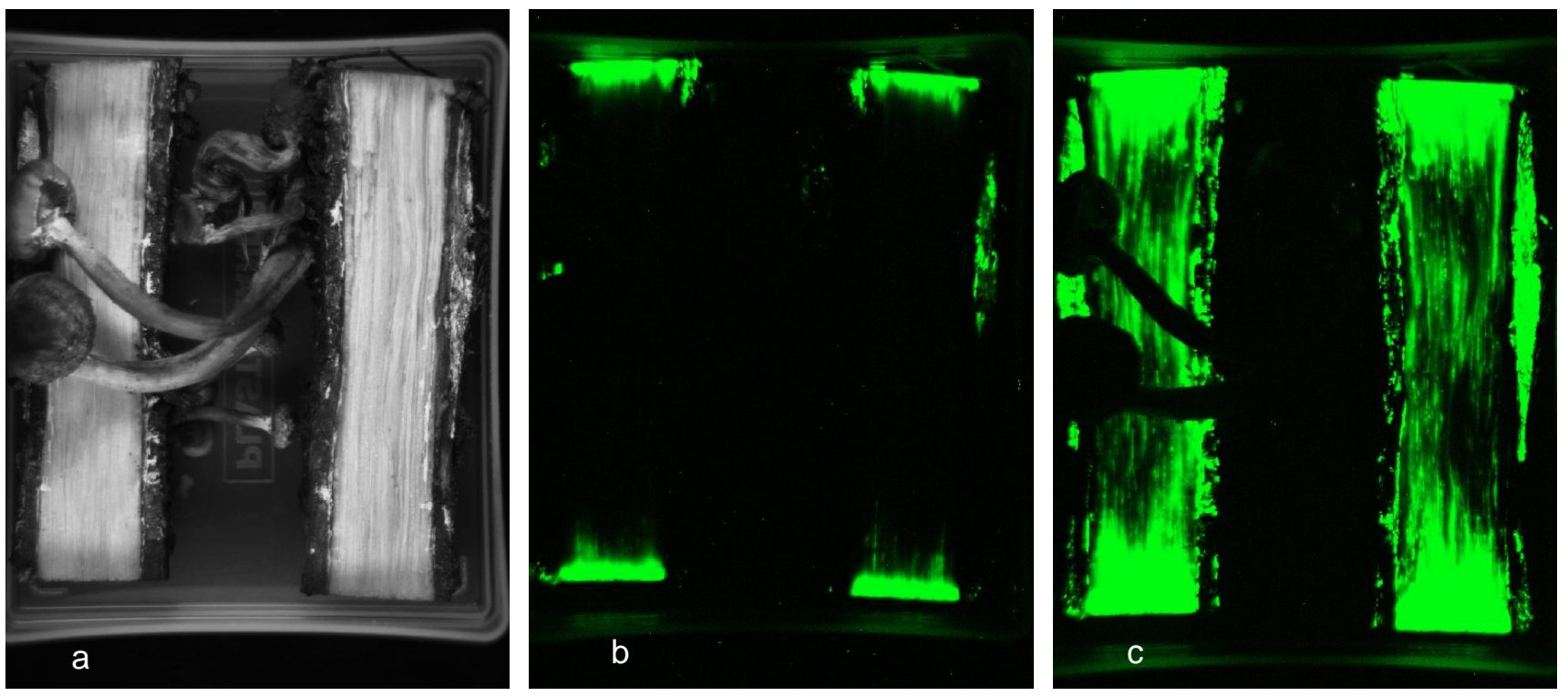

Fig. 2 - Luminescence of the sample at different time points after cleaving the wood: a. visible light. b. luminescence immediately after cleaving the wood. c. luminescence via $4 \mathrm{~h}$ after cleaving the wood. Images prepared using a GelDoc XR Imaging System. 


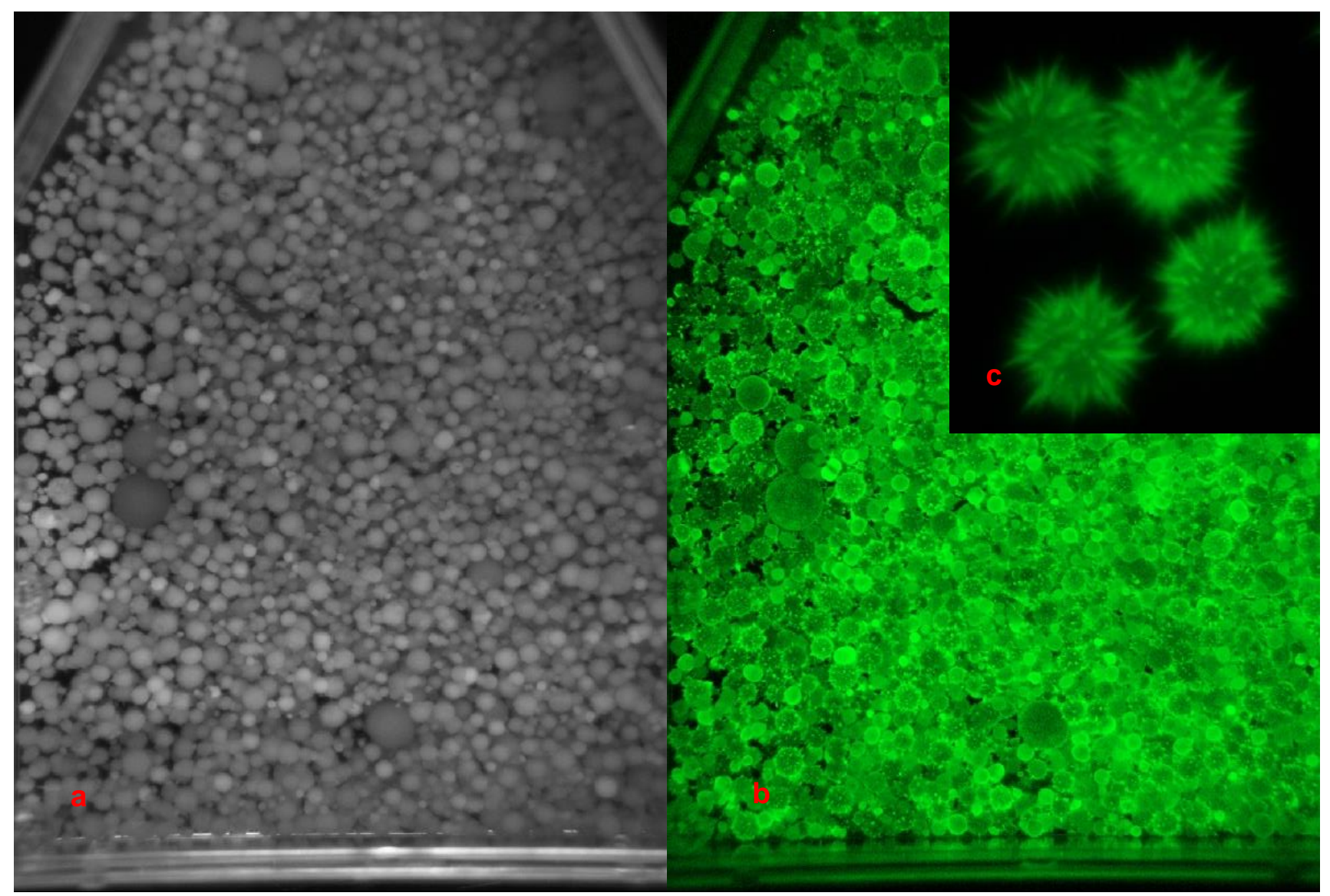

Fig. 3 - Appearance of A. borealis IBSO 2328 mycelium pellets: a. in visible light. b. luminescence of pellets. c. luminescence of individual pellets. Images prepared using a GelDoc XR Imaging System.

\section{Growth and luminescence of the fungus A. borealis in nature}

The appearance of nonluminous fruiting bodies of the fungus A. borealis growing in natural conditions on the natural substrate (wood) and the luminescence and changes in the mycelium luminescence intensity after cleaving the wood are shown in Fig. 1 and Fig. 2.

\section{A. borealis mycelium pellets - biomass for preparing cold extract}

Fig. 3 shows the appearance of $A$. borealis IBSO 2328 mycelium pellets after incubation in deionized water with the air constantly bubbled through the water.

\section{Luminescence of the cold extract from A. borealis mycelium}

Figures 4 and 5 show the intensity and dynamics of light emission by the cold extract from A. borealis IBSO 2328 mycelium with the sequentially supplemented of NADPH and the hot extract from P. squarrosa fruiting bodies (Fig. 4), and NADPH and the hot extract from A. borealis fruiting bodies (Fig. 5).

\section{Luminescence of the cold extract from A. borealis fruiting bodies}

Intensity and dynamics of light emission by the cold extract from A. borealis fruiting bodies sequentially supplemented with NADPH and the hot extract from P. squarrosa fruiting bodies are shown in Fig. 6. 


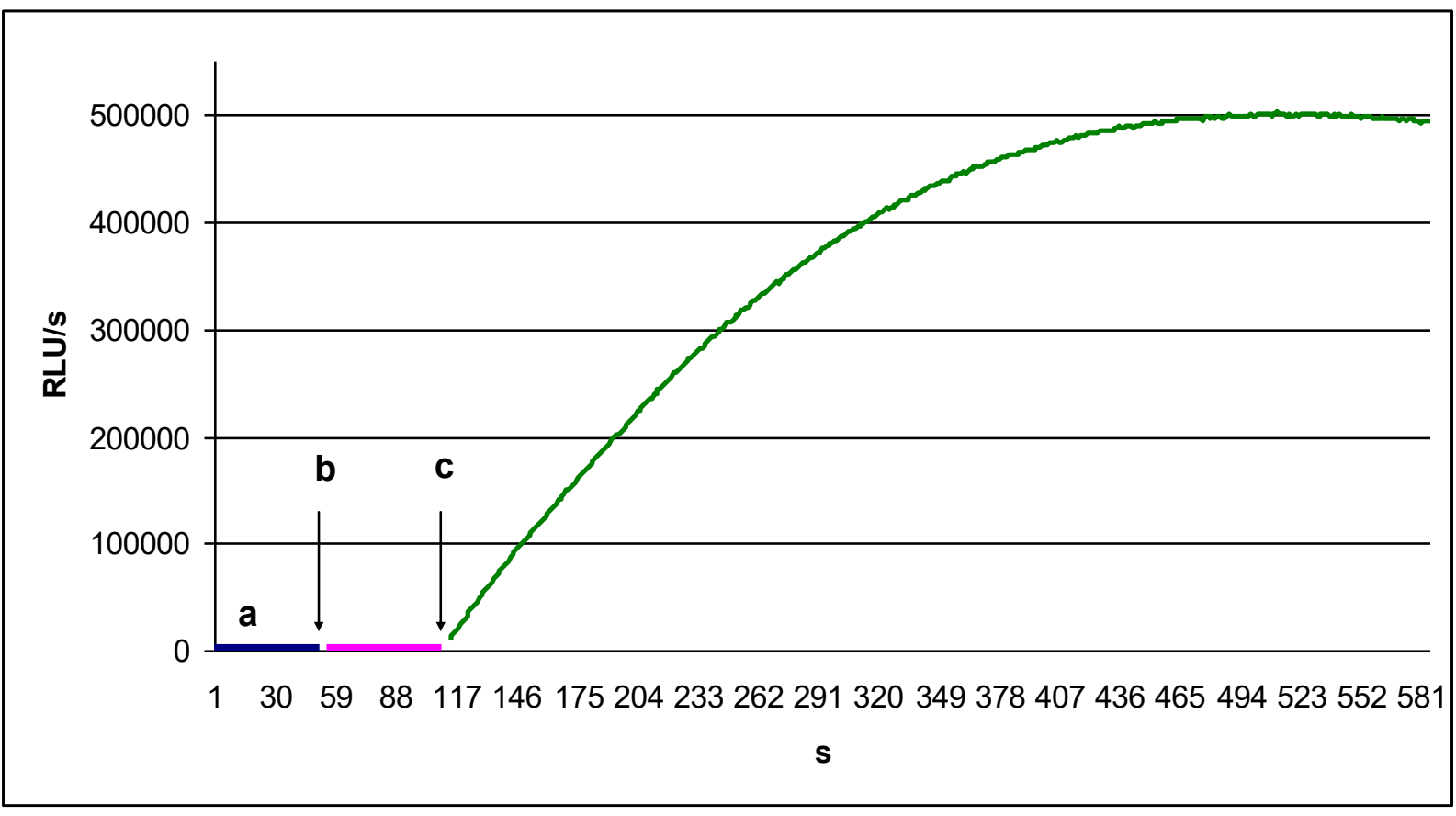

Fig. 4 - Luminescence: a. at the time when $200 \mu \mathrm{l}$ of the cold extract was poured into the microtube. b. after addition of $5 \mu 1$ of $10 \mathrm{mM}$ NADPH. c. after addition of $5 \mu \mathrm{l}$ of the hot extract from $P$. squarrosa fruiting bodies. Measurements were done by using Glomax® 20/20.

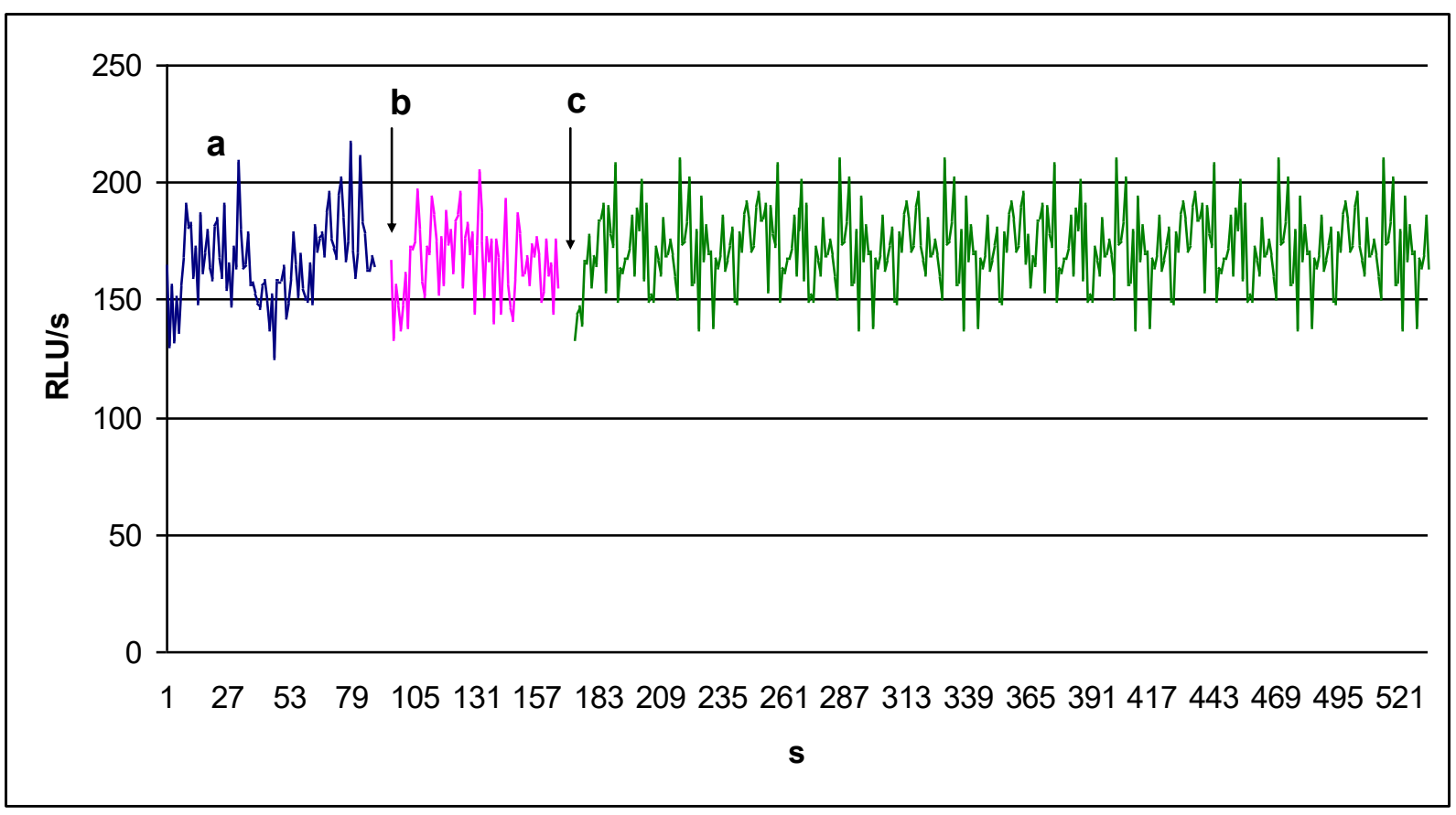

Fig. 5 - Luminescence: a. at the time when $200 \mu \mathrm{l}$ of the cold extract was poured into the microtube. b. after addition of $5 \mu \mathrm{l}$ of $10 \mathrm{mM}$ NADPH. c. after addition of $5 \mu \mathrm{l}$ of the hot extract from A. borealis fruiting bodies. Measurements were done by using Glomax ${ }^{\circledR}$ 20/20. 


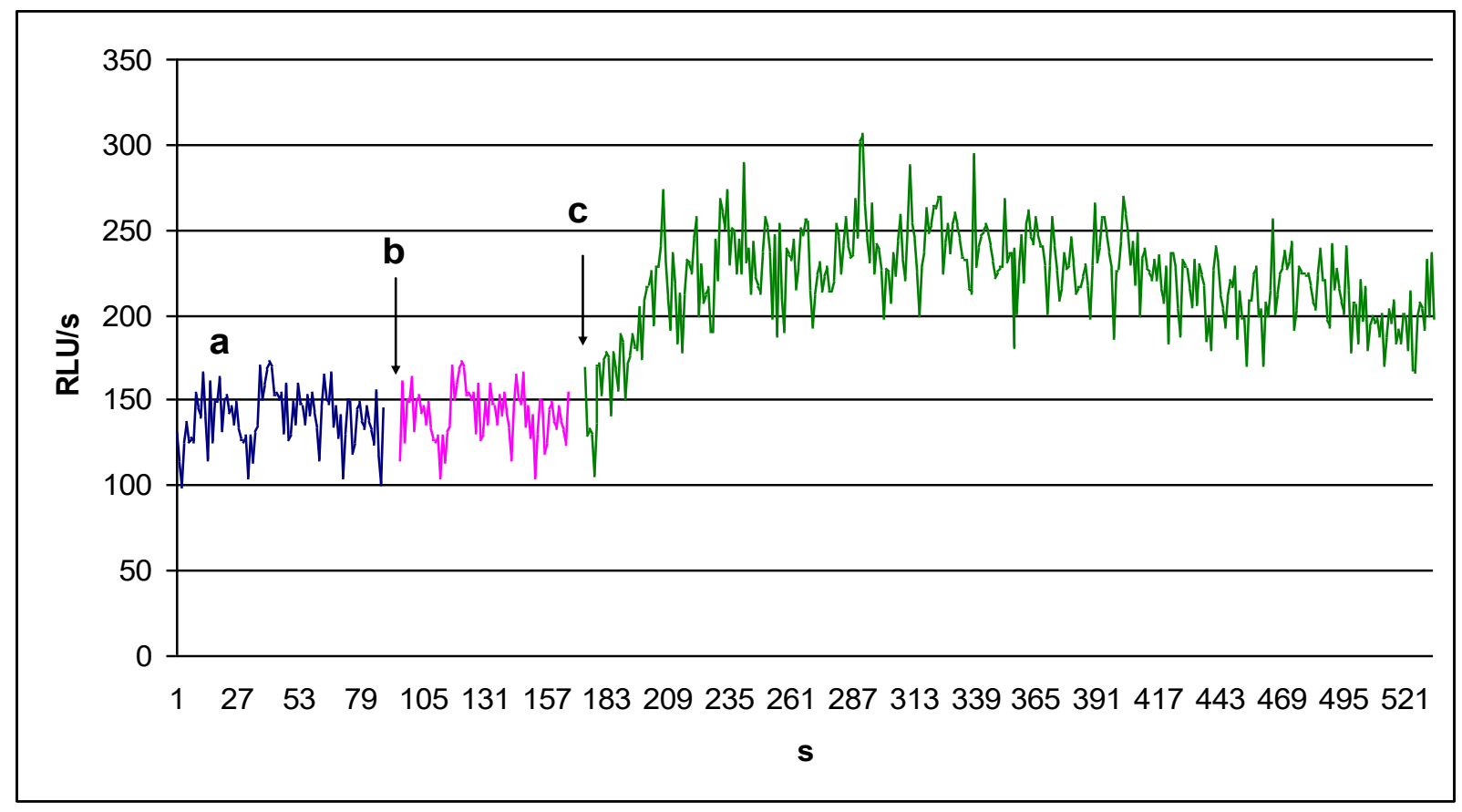

Fig. 6 - Luminescence: a. at the time when $200 \mu \mathrm{l}$ of the cold extract was poured into the microtube. b. after addition of $5 \mu 1$ of $10 \mathrm{mM}$ NADPH. c. after addition of $5 \mu \mathrm{l}$ of the hot extract from $P$. squarrosa fruiting bodies. Measurements were done by using Glomax ${ }^{\circledR}$ 20/20.

\section{Discussion}

\section{Growth and luminescence of the fungus A. borealis in nature}

Results of detection of luminescent zones (Fig. 1, 2) show that the fruiting bodies $A$. borealis and the wood into which mycelium has grown normally do not emit light. The luminescent signal from the wood is generated after some time, reaching its maximum in $4-6$ hours after external impact: mechanical injury to the mycelium, changes in the moisture content, and an increase in concentration of oxygen, which is necessary for the luminescent reaction to occur. The GelDoc XR Imaging System did not detect light emission by the fruiting bodies of any of the Armillaria strains used in our study, but the Glomax ${ }^{\circledR}$ 20/20 luminometer, which is more sensitive, can detect a weak level of chemiluminescence from the fruiting bodies. However, investigation of chemiluminescence which is typical to the fruiting bodies of nonluminous fungi (Gitelson et al. 2012, Van et al. $2013 \mathrm{a}, \mathrm{b}$ ) is outside the scope of the present study.

\section{A. borealis mycelium pellets - biomass for preparing cold extract}

The mycelium of $A$. borealis IBSO 2328 grown in laboratory on agar media (data not included) and in liquid culture (Fig. 3) exhibited strong luminescence. A. borealis cultivated in liquid nutrient medium produced large amounts of the biomass of mycelium pellets - starting material for preparing cold extracts, which contain enzymes of the luminescent system in the fungus. The pure culture IBSO 2328 was isolated from the nonluminous fruiting body of $A$. borealis. Thus, the genetic apparatus of the fruiting bodies of Armillaria must contain genes responsible for the synthesis of the enzymes and substrate of the luminescent reaction. Hence, we can propose three possible reasons why the fruiting bodies do not emit light. First, in the fruiting bodies these genes may be repressed and no components of the luminescent reaction (enzymes and substrate) can be synthesized. Second, one of the components of the luminescent reaction may not be synthesized. Third, the components of the luminescent reaction may be synthesized, but metabolites produced during growth of fruiting bodies may inhibit them. 


\section{Luminescence of the cold extract from A. borealis mycelium}

At the first step of experiments we examined luminescent properties of the cold extract from A. borealis IBSO 2328 mycelium pellets (Fig. 4). As a substrate analog we used the hot extract from $P$. squarrosa fruiting bodies, since it is known that it causes an increase the luminescent signal of cold extracts from some fungi (Purtov et al., 2015, Puzyr et al., 2016). The data shown in Fig. 4 suggest that the cold extract from IBSO 2328 mycelium pellets was nonluminous, and the addition of NADPH did not induce luminescence. The subsequent addition of the hot extract from $P$. squarrosa fruiting bodies induced a long-lasting luminescent signal with the light emission amplitude reaching $5 \times 10^{5} \mathrm{RLU} / \mathrm{sec}$. Hence, the cold extract from A. borealis IBSO 2328 mycelium contained enzymes, but did not contain NADPH and active substrate of the luminescent reaction.

As our experiment showed that addition of NADPH and the hot extract from the $P$. squarrosa fruiting bodies to the cold extract from A. borealis IBSO 2328 mycelium induced development of luminescence, we carried out an experiment with hot extracts from $A$. borealis fruiting bodies used as substrate. The experiment showed (Fig. 5) that after NADPH and the hot extract from A. borealis fruiting bodies were added to the cold extract from IBSO 2328 mycelium the luminescent signal remained unchanged and corresponded to the level of the background noise of the Glomax ${ }^{\circledR}$ 20/20 luminometer. Similar results were obtained in experiments with hot extracts from several Armillaria sp. fruiting bodies. Thus, hot extracts of the fruiting bodies of basidiomycetes of the genus Armillaria contained no substrate of the luminescent reaction.

\section{Luminescence of the cold extract from A. borealis fruiting bodies}

Our experiments showed (Fig. 6) that addition of NADPH and the hot extract from $P$. squarrosa fruiting bodies to the cold extracts from A. borealis fruiting bodies did not cause any significant changes in luminescence. The luminescent signal was insignificantly stronger than the background noise of the Glomax ${ }^{\circledR}$ 20/20 luminometer: the intensities of the light signals were 220 - $240 \mathrm{RLU} / \mathrm{sec}$ and $130-150 \mathrm{RLU} / \mathrm{sec}$, respectively. Similar data were obtained in experiments with cold extracts from Armillaria sp. fruiting bodies.

This may suggest that cold extracts from the fruiting bodies of A. borealis and Armillaria sp. contained very low amounts of enzymes in the luminescent reaction. However, as the differences between the intensity of the luminescent signal and the level of the background noise of the measuring device were extremely small, we can not be completely sure that these differences were significant. Thus, the effect observed in our study cannot be considered as a sufficiently convincing proof that cold extracts from the fruiting bodies of the genus Armillaria contain the enzymes of luminescent reaction.

Analysis of the data (Fig. 4 -6) currently suggests the following biochemical causes of the absence of visible luminescence in fruiting bodies of basidiomycetes of the genus Armillaria: (i) the absence of the substrate of the luminescent reaction and (ii) - the absence or very low amounts of the enzymes of luminescent reaction.

\section{Acknowledgements}

This work was supported by the state budget allocated to the fundamental research at the Russian Academy of Sciences (project no. 0360-2014-0006) and Program No.II.2 «Integration and Development» of the Siberian Branch of the Russian Academy of Sciences (project no. 03602015-0003).

\section{References}

Bondar VS, Rodicheva EK, Medvedeva SE, Tyulkova NA, Tyaglik AB, Shpak BA, Gitelson JI. 2013 - On the mechanism of luminescence of the fungus Neonothopanus nambi. Doklady Biochemistry and Biophysics 449, 80-83.

Brandl H. 2011 - Luminescent wood in coal and ore mines. Fungi 4, 5-9. 
Chew ALC, Desjardin DE, Tan YS, Musa MY, Sabaratnam V. 2015 - Bioluminescent fungi from Peninsular Malaysia - a taxonomic and phylogenetic overview. Fungal Diversity 70, 149187.

Desjardin DE, Capelari M, Stevani CV. 2007 - Bioluminescent Mycena species from Sao Paulo, Brazil. Mycologia 99, 317-331.

Desjardin DE, Oliveira AG, Stevani CV. 2008 - Fungi bioluminescence revisited. Photochemical \& Photobiological Sciences 7, 170-182.

Gitelson JI, Bondar VS, Medvedeva SE, Rodicheva EK, Vydryakova GA. 2012 Chemiluminescent emission of tissues of fruit bodies of higher fungi. Doklady Biochemistry and Biophysics 443, 105-108.

Harvey EN. 1952 - Bioluminescence. New York: Academic Press.

Harvey EN. 1957 - A history of luminescence from the earliest times until 1900. Baltimore, Maryland: J.H. Furst Company.

Johnson FH, Haneda Y. 1966 - Bioluminescence in Progress. Princeton: Princeton University Press.

Medvedeva SE, Artemenko KS, Krivosheenko AA, Rusinova AG, Rodicheva EK, Puzyr AP, Bondar VS. 2014 - Growth and light emission of luminous basidiomycetes cultivated on solid media and in submerged culture. Mycosphere 5, 565-577.

Mihail JD, Bruhn JN. 2007 - Bioluminescence is widespread within the Kingdom of fungi. Opera Mycologica 1, 28-33.

Mihail JD. 2013 - Comparative bioluminescence dynamics among multiple Armillaria gallica, A. mellea, and A. tabescens. Fungal Biology 117, 202-210.

Mihail JD. 2015 - Bioluminescence patterns among North American Armillaria species. Fungal Biology 119, 528-537.

Mogilnaya OA, Ronzhin NO, Bondar VS. 2016 - Comparative evaluation of total peroxidase and catalase activities during light emission of luminous fungus Neonothopanus nambi. Mycosphere 7, 499-510.

Mogilnaya OA, Ronzhin NO, Medvedeva SE, Bondar VS. 2015 - Total peroxidase and catalase activity of luminous basidiomycetes Armillaria borealis and Neonothopanus nambi in comparison with the level of light emission. Applied Biochemistry and Microbiology 51, 419-424.

Mori K, Kojima S, Maki S, Hirano T, Niwa H. 2011 - Bioluminescence characteristics of the fruiting body of Mycena chlorophos. Luminescence 26, 604-610.

Oliveira AG, Stevani CV, Waldenmaier HE, Viviani V, Emerson JM, Loros JJ, Dunlap JC. 2015 Circadian control sheds light on fungal bioluminescence. Current Biology 25, 1-5.

Purtov KV, Petushkov VN, Baranov MS, Mineev KS, Rodionova NS, Kaskova ZM, Tsarkova AS, Petunin AI, Bondar VS, Rodicheva EK, Medvedeva SE, Oba Yuichi, Oba Yumiko, Arseniev AS, Lukyanov S, Gitelson JI, Yampolsky IV. 2015 - The chemical basis of fungal bioluminescence. Angewandte Chemie International Edition 54, 8124-8128.

Puzyr AP, Medvedeva SE, Bondar VS. 2016 - The use of glowing wood as a source of luminescent culture of fungus mycelium. Mycosphere 7, 1-17.

Shimomura O. 2006 - Bioluminescence: chemical principles and methods. Singapore: World Scientifc Publishing Co. Pte. Ltd. Pp. 266-300.

Teranishi K. 2016a - Localization of the bioluminescence system in the pileus of Mycena chlorophos. Luminescence 31, 594-599.

Teranishi K. 2016b - trans-p-Hydroxycinnamic acid as a bioluminescence-activating component in the pileus of the luminous fungus Mycena chlorophos. Tetrahedron 72, 726-733.

Van DT, Manukovsky NS, Gitelson JI. 2013a - Chemiluminescence of mushrooms cultivated in Vietnam. Doklady Biochemistry and Biophysics 448, 13-14.

Van DT, Manukovsky NS, Gitelson JI. 2013b - Chemiluminescence of basidiomycetes of the tropical forest of South Vietnam. Doklady Biochemistry and Biophysics 450, 140-142. 
Van DT. 2009 - Physiological and cultural properties of the luminous fungus Omphalotus af. illudent. Journal of Siberian Federal University. Biology 2(2), 157-171.

Vydryakova GA, Psurtseva NV, Belova NV, Pashenova NV, Gitelson JI. 2009 - Luminous mushrooms and prospects of their use. Mikologia \& Fitopatologia 43, 369-376.

Vydryakova GA, Van DT, Shoukouhi P, Psurtseva NV, Bissett J. 2012 - Intergenomic and intragenomic ITS sequence heterogeneity in Neonothopanus nambi (Agaricales) from Vietnam. Mycology 3, 89-99.

Wassink EC. 1978 - Luminescence in fungi. In: Bioluminescence in Action. London: Academic Press. Pp. 171-197. 